\title{
Tubal ectopic pregnancy on a patient with two previous consecutive laparoscopic tubal sterilisation techniques
}

\author{
Costas Panayotidis • Richard Husicka
}

Received: 20 October 2012 / Accepted: 31 December 2012 /Published online: 18 January 2013

(C) Springer-Verlag Berlin Heidelberg 2013

\section{Introduction}

The article discusses the challenges of laparoscopic management of failed sterilisation techniques, the difficulty of further laparoscopic tubal sterilisation techniques using diathermy and the limitations of future hysteroscopic sterilisation when the proximal tubal end is missing.

Case study

A 35-year-old patient gravida 6 para 3 had an uncomplicated laparoscopic sterilisation where two Filshie clips were applied on both tubes (Fig. 1). Four years later, she has been referred to the gynaecological clinic with 18 months history of deteriorating abdominal pain, described as lower pelvic dull ache, predominantly on the right iliac fossa side. The patient did not have dysmenorrhoea or other clinical symptoms suggestive of endometriosis

Diagnostic laparoscopy was performed and three migrated clips, all attached curiously together, where removed from the right upper part of the vesico-uterine peritoneum (Fig. 2). At this point, both the right and left cornual ends of uterus were of normal appearance with a very short proximal tubal end. The right tube had an impregnation of the previously applied clips presenting as a very small intermediate part and a longer distal part (Fig. 3). The laparoscopy did not detect any other pelvic abnormality, no endometriosis, or adhesions were depicted. The fourth clip was not detected despite a thorough inspection of the pelvis and the left distal tubal end was intact. Bipolar diathermy at $20 \mathrm{~W}$

C. Panayotidis $(\bowtie) \cdot$ R. Husicka

Gynecology, Withybush General Hospital,

Haverfordwest, Wales, UK

e-mail: costapan@hotmail.com was applied on the remaining proximal tubal ends at their whole length in order to secure tubal blockage and the right intermediate tubal part was removed simultaneously (Fig. 4). Neither was possible to apply any type of sterilisation clip on such short tubal ends. Any attempt to excise the proximal tubal ends with scissors/monopolar energy would involve excision of part of the uterine cornua and hence could lead to complications and conversion to laparotomy. Patient had an excellent recovery and her previously described pains were quickly resolved.

Seven months later, she had an unexpectedly positive pregnancy test after missing her period for more than a month. Although the patient was asymptomatic, she came to the early pregnancy unit for a transvaginal ultrasound scan which was strongly suggestive of right tubal ectopic pregnancy $(4 \mathrm{~cm}$ "doughnut" like structure in the right adnexal area, demarkable from the ovary) and her serum beta HCG was 22,959 IU/1.

Patient was fully consented for diagnostic laparoscopy and bilateral salpingectomy. The diagnostic laparoscopy confirmed the ectopic pregnancy in the remained distal right end of fallopian tube which was treated with a straight forward salpingectomy using Ligasure ${ }^{\circledR}$ (Fig. 5). The fourth clip was then detected parallel the left distal fallopian tube, imbedded in dense adhesions and after adhesiolysis, a left salpingectomy was performed. Furthermore, a blue methylene dye test was performed pointing through the proximal tubal transparency; the lumen of the cornual part of both tubes without spillage (Fig. 6). Decision was made to proceed with bipolar diathermy $(20 \mathrm{~W})$ on the proximal ends of previous fallopian tubes.

Patient had a good postoperative recovery and she had a hysterosalpingography (Fig. 7) which confirmed blockage of the cornual/proximal tubal origins with prompt follow up in outpatient clinic. The patient was thoroughly informed 
Fig. 1 Two Filshie clips were applied on both tubes
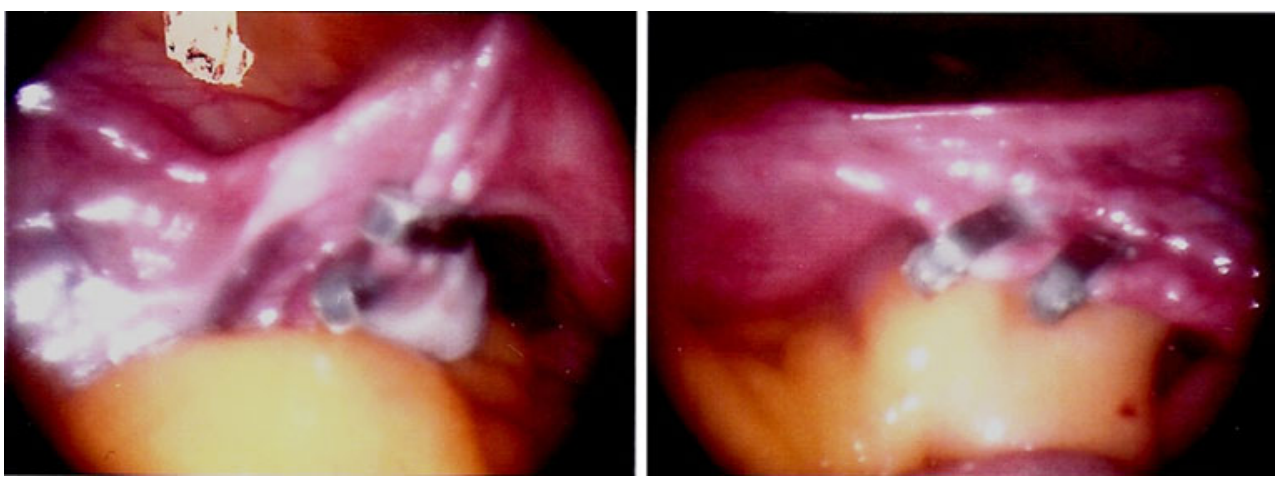

about the technical limitations to secure long-term complete tubal occlusion and she was offered Mirena coil which she accepted.

\section{Discussion}

This case report may generate many questions about failed sterilisation techniques which need to be discussed, particularly about appropriate usage of dye test/or hysterosalpingography in cases of failed or complicated sterilisation, how important is the consenting for the procedure and how big role can play laparoscopic surgical experience. It is important to emphasise that this patient was under the care of gynaecologist with different level of experience in laparoscopic sterilisation techniques.

Failed sterilisation techniques have been already described in the literature [1]. Some of these cases have been complicated with ectopic pregnancies; other cases needed termination of intrauterine pregnancy or rarely patient decided to keep the pregnancy. Patients can be in great discomfort clinically and psychologically and they need adequate support [2]. However, this case report is in our best knowledge the first one that reports an ectopic tubal unruptured pregnancy after two consecutive sterilisation surgeries with two different techniques [3]. Filshie sterilisation technique is the most popular in UK [4] and following the Royal College of Obstetricians and Gynaecologists guidelines, the failure rate is approximately $1 / 200$. In case of diathermy using bipolar energy for tubal sterilisation, the failure rate is difficult to estimate as there are no published data. In the past, monopolar diathermy on the tubes was used with both higher failure and intraoperative complications rates. In our best knowledge, there are no studies comparing Filshie clip application and bipolar diathermy and their sterilisation failure rates [5].

In our case, the patient needed a diagnostic laparoscopy due to her persistent pelvic pains and three migrated clips (curiously attached all together) were removed successfully. During the first laparoscopic sterilisation, two clips were applied on each tube; however, at the second laparoscopy 4 years later, the remaining proximal end of each tube was very short not allowing any further clip application.

Some will argue that intraoperative dye test should always be done after retrieval of migrated clips in order to obtain macroscopic evidence of tubal blockage and consequently to avoid any surgical interference with the proximal tubes. The potential risk of weak proximal tubal fibrosis in this case with future tubal recanalisation is impossible to evaluate with dye test or hysterosalpingography. A dye test, in these cases of extremely short proximal tubal ends, may not reveal unblocked tubes (as it was confirmed during the third laparoscopy of this patient). Due to the increased intrauterine pressure generated during the dye test, there is a risk of "flush" and partial or complete future desobstruction of the thin fibrotic plug that resulted from the previous migrated Filshie clip. For these reasons in this particular case, it was thought beneficial to diathermise the proximal
Fig. 2 Three migrated clips adherent together on the vesicouterine pouch
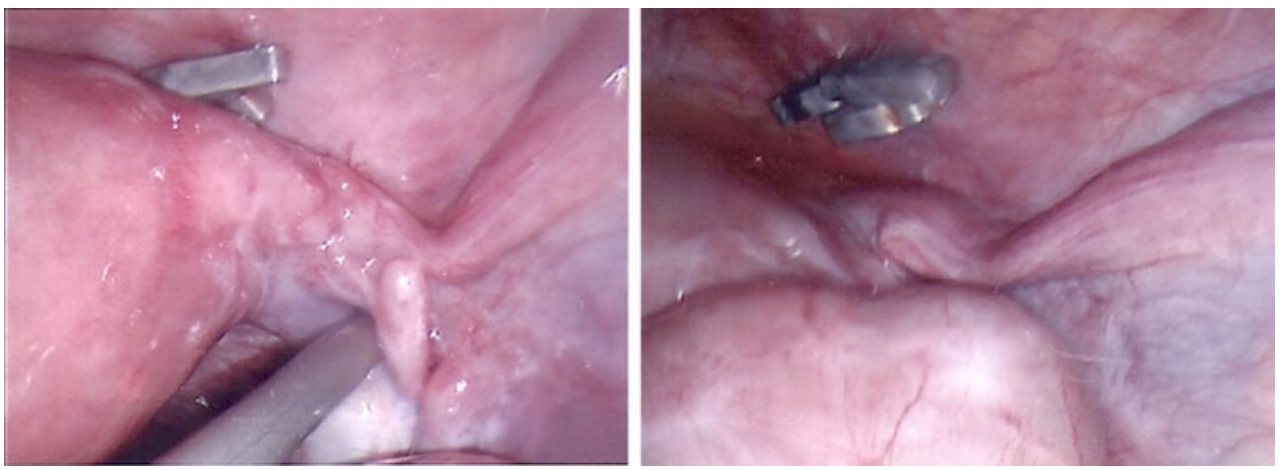


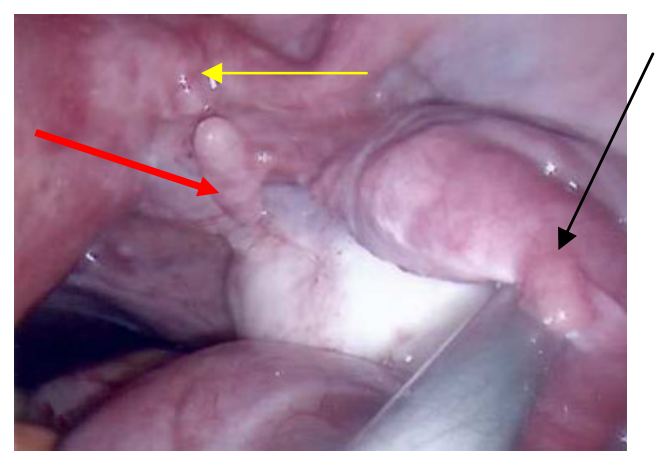

Fig. 3 The right fallopian tube had an "impregnation" of the previously applied Filshie clips resulting to a very small intermediate part (red arrow) and a longer distal one (black arrow). Yellow arrow point the short proximal (cornual) part of the fallopian tube

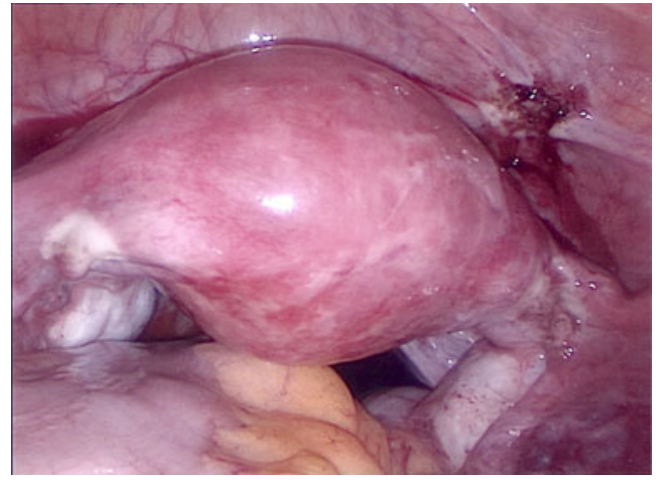

Fig. 4 The remained proximal tubal ends were electro-coagulated after retrieval of the migrated clips

tubes during the second laparoscopy as the proximal tubal ends were very short from both sides and far away from the tubal clip application point (intermediate part of the tube).

Others will argue that in case of bipolar diathermy the technique needs to secure adequate placement of the bipolar grasper on the surface and periphery of the tubal end. This

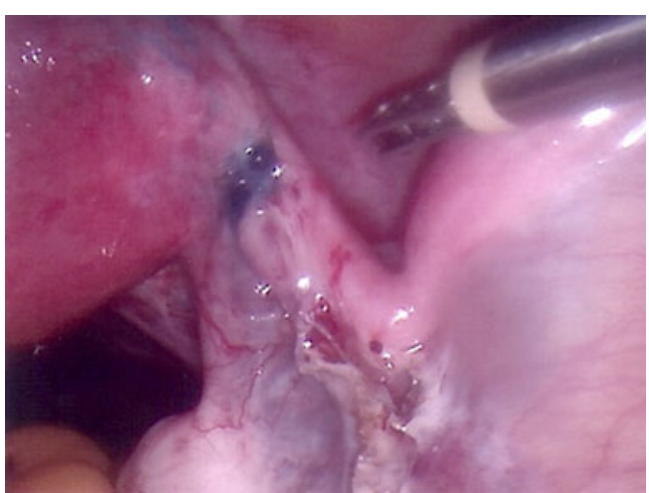

Fig. 6 Dye test pointing the lumen of the cornual part of the right fallopian tube without spillage

coagulating consequence of diathermy is difficult to evaluate as the fibrotic effect will depend on the amount of energy applied and how long this was applied for, on the thickness of the proximal tube and also it will depend on the nature of the coagulated tissues and finally the endogenous potential of healing.

Laparoscopic stitching of the uterine cornual end is difficult because needs expertise to avoid uterine trauma and bleeding. The surgical laparoscopic experience and adequate laparoscopic equipment for such operative laparoscopy were not available for the second and third procedure. The consent of this patient did not include conversion to laparotomy for salpingectomy (proximal ends) but only conversion to laparotomy in case of laparoscopic entry technique complications.

With the third laparoscopy, the surgical challenge was first to treat successfully the unruptured tubal ectopic pregnancy with prompt uncomplicated salpingectomies on both remaining distal tubes and secondary to deal with the even shorter cornual tubal ends (remainders) in order to avoid another failure.

A secondary electrocoagulation applied on a larger area at the tubal-cornual origin was performed with the plan to review the patient with a hysterosalpingography 2 months
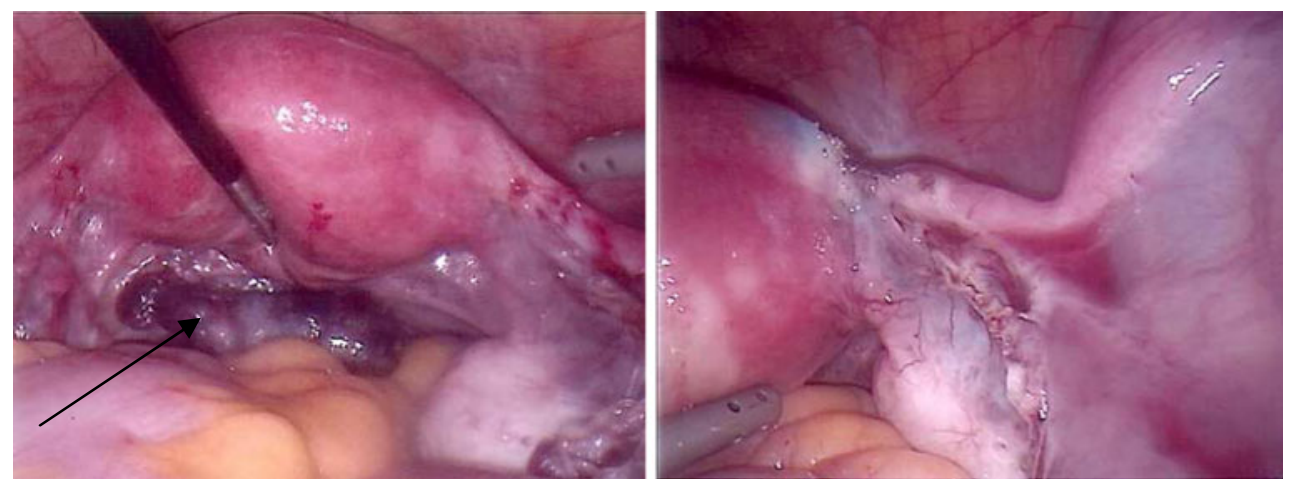

Fig. 5 Salpingectomy for unruptured ectopic. Black arrow tubal ectopic unruptured pregnancy after salpingectomy parked in the pouch of Douglas before retrieval 


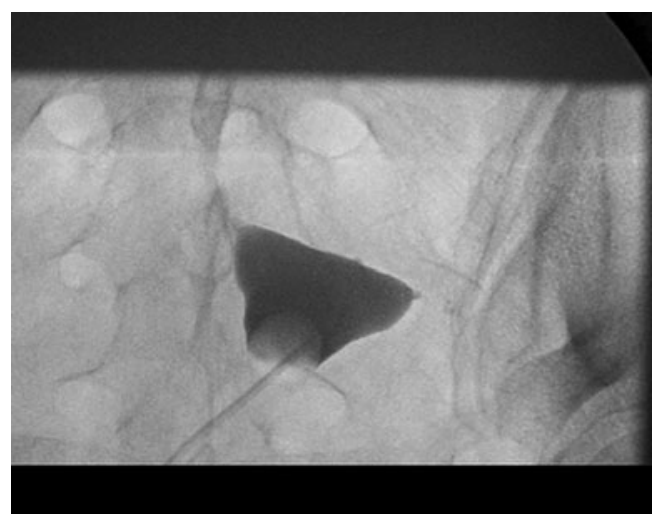

Fig. 7 Hysterosalpingography with bilateral cornual blockage

postoperatively in order to demonstrate obstruction. However, even if the hysterosalpingography can show an obstruction it is uncertain for how long this will be maintained and how to follow up the patient.

Hysteroscopic sterilisation is not possible as there is no proximal tubal end long enough to secure permanent and uncomplicated insert placement. Hysteroscopic usage of intrafallopian "glue" for obstruction is not yet available. Further extensive laparoscopic excisions of the uterine cornual ends cannot guarantee complete obstruction either.

The future management options were discussed with the patient in the postroperative follow up and she opted for MIRENA IUS ${ }^{\circledR}$ rather other long-term contraceptive method or further surgery with laparotomy (cornual excision or hysterectomy).

Some questions and uncertainties could be highlighted with this case report. We do not know what could be the physiological healing history of the remaining proximal tube in case of migrated Filshie clips and so whether a recanalisation is a rare but unavoidable risk or whether there is an increased likelihood of recanalisation after usage of diathermy. It could be assumed that all would depend on the length and consistency of the fibrosis which is maintained at the most distal part of the proximal remaining tube. Most of surgeons use currently only one Filshie clip adequately applied on each tube. However in our case, two clips were applied and the ischemia and scar formation could be different than with 1 clip application. In our case, also all four clips migrated. Perhaps the usage of two clips on each tube might have created more tubal ischemia and hence higher risk of clip migration in long term. The ischemic tissue might become weaker in time and clips might not maintain in the appropriate position on the tubal isthmus.

\section{Conclusion}

In conclusion, most of the published reports refer to medicolegal aspect [2] of the cases with failed sterilisation techniques, especially those that occurred after a short period of time post operatively. Adequate laparoscopic technique and placement of Filshie clips minimise the risk of migration and possible recanalisation of the proximal tubal ends. There is little evidence to describe the risks of failed sterilisation and its correlation with migration of clips in the long term. In case of secondary laparoscopic correction of failed sterilisation, adequate consent needs to be done. Such consent should include the discussion about limits of preoperative and postoperative imaging techniques to confirm long term tubal obstruction, usage of long-term contraception methods and in case that the laparoscopic approach can not secure tubal blockage, to complete the bilateral salpingectomy (proximal-distal ends) with laparotomy.

\section{References}

1. Awonuga AO, Imudia AN, Shavell VI, Berman J, Diamond MP, Puscheck EE (2009) Failed female sterilization: a review of pathogenesis and subsequent contraceptive options. J Reprod Med 54 (9):541-547

2. Varma R, Gupta JK (2007) Predicting negligence in female sterilization failure using time interval to sterilization failure: analysis of 131 cases. Hum Reprod 22(9):2437-2443

3. Ruminjo JK, Chabari C (1993) Twice-failed tubal ligation: a case report. East Afr Med J 70(8):528-530

4. Penfield AJ (2000) The Filshie clip for female sterilization: a review of world experience. Am J Obstet Gynecol 182(3):485-489

5. Lawrie TA, Nardin JM, Kulier R, Boulvain M (2011) Techniques for the interruption of tubal patency for female sterilization. Cochrane Data Systematic Review. doi:10.1002/14651858 